\author{
Herbert Gursky \\ Center for Astrophysics \\ Smithsonian Astrophysical Observatory/ \\ Harvard College Observatory \\ Cambridge, Massachusetts 01776 , \\ USA
}

\title{
INTRODUCTION
}

The fact that $\mathrm{x}$-ray astronomy could yield cosmological information was apparent in the earliest rocket flights carried out in 1962 and 1963 (Giacconi et al, 1962, Bowyer et al,1963). These flights established that diffuse radiation was present around the sky; indeed one of the first theoretical papers commenting on the $x$-ray results was by Fred Hoyle (1963) showing that the observed background flux was below that predicted for the hot, steady-state universe. A few years later, the first discrete source was credibly identified with an external galaxy (Byram, et a1. 1966).

Figure 1 illustrates the primary data that provides the basis for an optimistic view that cosmological information is present. The figure contains a single day of data from the Uhuru satellite taken along a $5^{\circ}$ great circle band, passing near the galactic pole. The data show two prominent extragalactic objects, the Virgo cluster and Centaurus $A$; equally distinct is the high radiation level present at all galactic latitudes with no perceptible variation in intensity. This radiation is the diffuse $x$-ray background and in the Uhuru detectors with $\sim 25 \mathrm{deg}^{2}$ solid angle dominates the appearance of the data.

It is now known that the diffuse radiation is isotropic and free of small scale fluctuations with a high degree of precision. It is also known that the superposition of known extragalactic $x-r a y$ sources cannot account for the radiation. Thus, cosmological information should be available on several counts.

1. The diffuse radiation must be originating at $z>1$ and may be as remote as $z \sim 10$. It may be revealing conditions in the intergalactic medium that are not otherwise known.

2. The $x$-ray emission from individual extragalactic sources may yield information about galaxies or other discrete sources bearing on 
the large scale structure of the universe, the evolution of cosmic objects or the chemistry of the universe.

In this paper, I will briefly review the present state of understanding of extragalactic x-ray astronomy. I will present what little cosmological information we have obtained to date and I will discuss how and possibly where new information of this kind may emerge. I am going on the presumption that the key areas are the nature of the diffuse $x$-ray background and of the discrete $x$-ray sources. There have been recent reviews of extragalactic astronomy by Horstman, Cavallo and Moretti-Horstman (1975), Gursky and Schwartz (1977), and Culhane (1977).

\section{THE DIFFUSE X-RAY BACKGROUND}

The data shown in Figure 1 are in the energy range 2-10 kev. There is also a strong component present below one kev that for a time was believed to be extragalactic; however that component is strongly correlated with respect to the Milky Way and it is not evident what fraction originates outside the Galaxy. There is background radiation extending to high energies as seen in Figure 2, which is from a compilation by Schwartz (1974). Apparently the radiation extends to $100 \mathrm{Mev}$; however information on its isotropy is meager above about 100 Kev. Cowsik and Kobetich (1972) demonstrated that the spectrum could not be matched to an inverse compton process, but that it could be tolerably well fit by a thermal spectrum with a temperature of $\sim 3 \times 10^{8} \mathrm{~K}$. This supports the idea that the radiation originates from a hot, intergalactic medium. The high energy data (above $\sim 100 \mathrm{kev}$ ) probably means that a second process is operating as well.

Fig. 1. Great circle distribution of $x$-ray counts seen by Uhuru. The upper plat $(x-2)$ is obtained by the $5 \times 5^{\circ}$ collimator and $\sim 80 \%$ of the observed flux (exclusive of point sources) originates in the diffuse

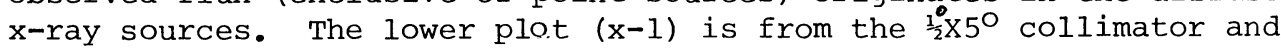
show a residual count rate of $\sim 7 \mathrm{ct} / \mathrm{sec}$ almost all of which can be attributed to non-x-ray events.

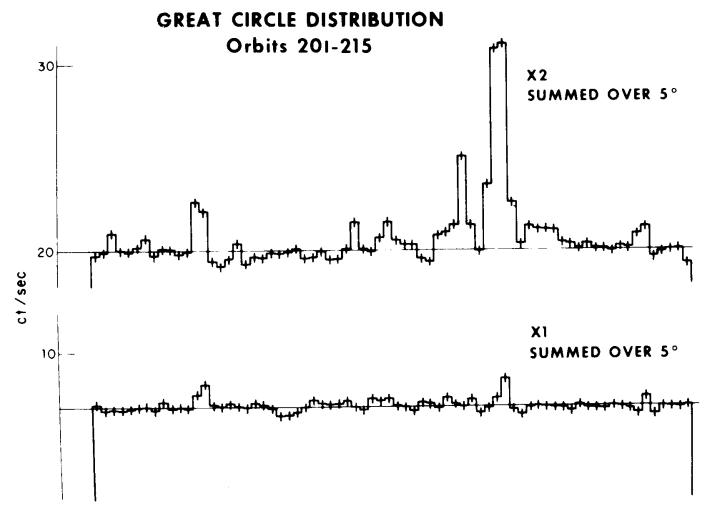


Fig. 2. The Spectrum of the Diffuse X-ray Background. The dashed line is an approximate fit of a thermal spectrum.

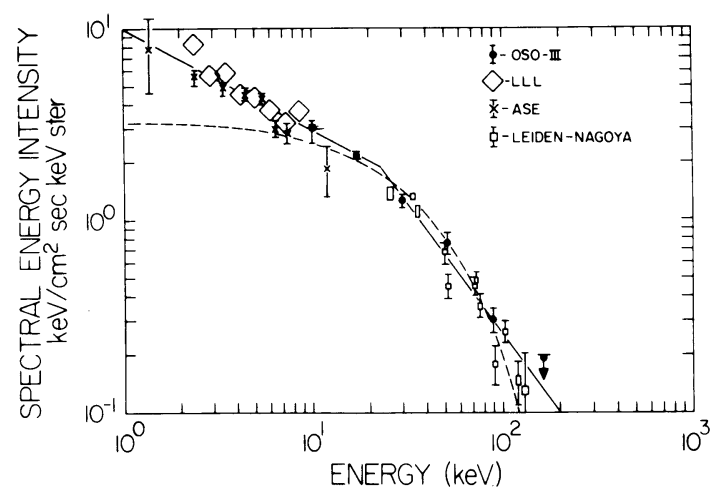

In the range of $2-40 \mathrm{kev}$ the large scale isotropy ( 200) is established to about 5\% (Schwartz, 1970). On a smaller scale, there are present fluctuations of $3.6 \%$ on a scale of $25 \mathrm{deg}^{2}$ as shown in Figure 3 (Schwartz, et al, 1976, Schwartz, 1977)。 The upper limit to the fluctuations is $4.6 \%$. This result is consistent with the logNlogs relation as derived from the $3 \mathrm{U}$ catalog to about a confidence 10\% level. The discrepancy, if any, is that the fluctuations are somewhat smaller than is predicted from the source counts. However, considering the possibility of systematic errors, the discrepancy is not to be taken as an indication of a deviation from a uniform distribution of discrete sources obeying a $3 / 2$ power law. The more recent logN-logs curve derived from the $4 \mathrm{U}$ catalog (Forman, et al, 1977) is consistent with the earlier results. The observation of fluctuations cannot be used to derive any information regarding the diffuse $x$-ray background. In particular, we cannot derive the fraction of background originating from a truly diffuse component. The reason for this is simply that fluctuations must result from the stronger of the unresolved sources; e.g., those only a factor of ten fainter than the ones presently observed. On the other hand, to make up the entire diffuse background, the observed logN logs curve must be extrapolated by $\sim 10^{3}$.

Some fraction of the diffuse $x$-ray background must originate in the fainter, unresolved sources. In fact, the observed high latitude Uhuru sources contribute about $2 \%$ of the background; i.e., a coarse, lower sensitivity experiment would measure the background flux about 2\% higher than did Uhuru. 
Fig. 3. Observations of the diffuse $x$-ray background with Uhuru (50x50 collimator) around a number of great circles in the sky. Points containing known sources are excluded. The point-to-point fluctuations are greater than can be accounted for by poisson statistics.

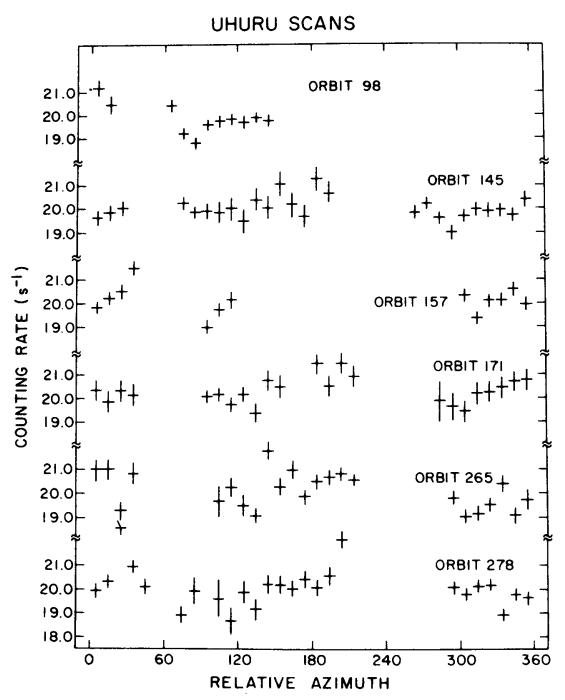

The diffuse radiation may originate in the intergalactic medium. As noted, the spectral distribution of the radiation is consistent with a thermal origin; if so, it is difficult to avoid the existence in the intergalactic gas of considerable mass, perhaps equivalent to $\Omega \sim 0.5$. However, the problem of heating the gas is not trivial and may comprise evidence of an energy producing stage in the evolution of matter that we have no evidence of now. (cf. Field and Perrenod, 1977)

\section{DISCRETE EXTRAGALACTIC X-RAY SOURCES}

The Third Uhuru catalog revealed the existence of a significant population of $x$-ray sources at high galactic latitudes comprising examples of normal galaxies, active galaxies and clusters of galaxies (Giacconi, et al, 1974). The most extensive kind of source was that associated with rich clusters of galaxies, as first discussed by Gursky, et al (1972), and extended by Kellogg, et al (1973). There was also revealed a class of sources that could not be identified with either prominent galaxies or quasars. These results have been extended in the Fourth Uhuru catalog (Forman, et al 1977) and the Ariel catalog (Cooke, et al 1977). These latter catalogs have revealed the Seyferts (Class I) to be prominent $\mathrm{X}$-ray sources as well. The present results reveal that in a region of the sky surveyed to a reasonable degree of completeness for both $\mathrm{x}$-ray sources and at least certain kind of Seyfert galaxies, 18 of 89 seyferts can be identified as x-ray sources (Tananbaum, et al 1977). For clusters of galaxies, the 
results are even more dramatic. Jones and Forman (1977) using combined Ariel 5 and Uhuru results, conclude that 33 Abell clusters within distance class 4 are $x$-ray sources. An additional 15 clusters, mostly in the south, are also found to be $x$-ray sources. As discussed by Tananbaum, et al (1977), the unseen seyferts will make up 10-20\% of the diffuse background, depending on the completeness of the present optical surveys. The clusters will contribute an additional $\sim 15 \%$ as discussed in this volume by Culhane. Thus, the identified sources fall short by a factor of 3 or more in accounting for the diffuse x-ray background.

More than half of the high latitude x-ray sources, presumably extragalactic, are still "unidentified". For example, in the Fourth Uhuru catalog, of $\sim 200$ sources for which $|\mathrm{b}|>20^{\circ}$, only 92 carry identifications. However, many of these identifications must be chance coincidences. Some will be Seyfert galaxies since the optical surveys for these objects are far from complete. Some of these may be galactic as indicated by the recent discovery of soft $x$-ray sources such as AM Hercules, but the distribution of the sources argues against a galactic origin. What are the unidentified sources and will they yield any cosmological information? The study by Bahcall et al (1975), of ten such sources, found at best two quasar candidates ( $m \sim 17.5$ ) and one peculiar galaxy. Their conclusion was that if the objects are associated with galaxies or blue stellar-like objects, the ratio of $\mathrm{x}$-ray to optical luminosity is at least 10 and possibly 100. Another clue, although a weak one, is found in the logN-logs curve which is consistent with a $3 / 2$ powers law, and the small scale fluctuations in the $x$-ray background which is consistent with a "naive" interpretation of the $\log N-\operatorname{logs}$ curve. These facts imply that the $\mathrm{x}$-ray sources are relatively nearby and not subject to strong evolution.

The unidentified sources may simply be telling us that active galaxies are far more extensive than we now believe and there is a very broad spread in the ratio of $x$-ray to optical luminosity of these objects just as is the radio to optical luminosity. If this is the case, at a minimum, a more complete study of extragalactic x-ray sources should significantly improve our understanding of the nature and evolution of active galaxies and since we know this extends to $z$ $\approx 3$ at least, provide some information on the early evolution of galaxies generally.

\section{CLUSTERS AND INTERGALACTIC GAS}

There are numerous indications of gas in between the galaxies, such as the morphology of double radio sources and the radio tails on certain galaxies, however the most extensive evidence for such gas has been the discovery of x-ray emission from clusters of galaxies. The emission correlates well with cluster richness and velocity dispersion and can be sensibly accounted for as resulting from a hot gas. The discovery of iron line emission from several of these cluster sources 
is the culminating evidence for a thermal origin (see Culhane's contribution to this volume for detailed discussion of $\mathrm{x}$-ray clusters).

Since the angular size of the emitting region has been found for several of the clusters, the mass of radiating gas is determined. It is typically $10^{13}-10^{14} \mathrm{~m} \circ$, namely comparable to the mass of the visible galaxies, but still a factor of ten below the virial mass for the clusters. Thus the gas does not resolve the problem of the discrepancy between the virial mass and the observed mass, but it does dramatically alter ones concept of a cluster of galaxies. Since there is so much mass in hot gas, one can no longer look at the galaxy distribution and make conclusions about the net gravitational field or the degree of relaxation in the cluster.

The fact that iron is present in that gas at near normal abundance is obviously of great significance. It is easy to say that the iron means that the gas is derived from the galaxies themselves. However with still 90\% of the matter yet to be accounted for, such an easy way out may be naive in the extreme.

Thus, the existence of hot gas in the clusters of galaxies is of obvious significance to the large scale structure of the Universe. One can for example imagine a society with only x-ray eyes to study the cosmos. Their view of the universe, at least as measured by "catalogs of clusters" (in their parlance "hot gas clouds") would not be terribly different from ours as derived visually. However, for these individuals, galaxies would be virtually absent.

Now we come to a remarkable discovery by Murray, et al (1977), albeit a somewhat tentative one. The Fourth Uhuru catalog revealed six instances in which several distant Abell clusters were within or near, the error box of a single x-ray source. Further examination revealed that these clusters comprised associations listed by Abell as superclusters. Murray, et al, using a rigorous definition of "supercluster", found three whose centers were coincident with x-ray sources. The result is statistically significant but still must be regarded as tentative since other possibilities arise immediately. For one, it is possible that a single cluster is the $x$-ray source. This cluster would have a luminosity of order $10^{46} \mathrm{erg} / \mathrm{sec}$, far in excess of what is seen for other clusters. The second possibility is that these are very luminous $x$-ray sources associated with a galaxy within one of the clusters. Also, the presence of the sources has yet be confirmed by independent measurements. The crucial data will be the angular size of the x-ray emitting regions. If the emission does originate in the supercluster, then the sources must show angular sizes of several degrees.

This could be a result of far reaching significance. For one the mass of gas is estimated by Murray, et al to be $\sim 10^{16} \mathrm{M}$ or far in excess of what is present in the visible galaxies in the region. Secondly, it makes more plausible the idea that hot gas fills the entire Universe, yielding up the diffuse $x$-ray background. Third, since 
there is so much gas, it can hardly be argued that it originates from galaxies. The gas could be primeval or it could result from a period of collapse prior to the one that led to the observed galaxies. Either way, a study of its chemical makeup, as is possible through $x$-ray spectroscopy, could reveal the conditions in a very massive component of the early universe. Finally, it provides more reality to the concept of supercluster; in fact the supercluster is the gas cloud since that is where the mass is。

\section{FUTURE PROSPECTS}

So far, only a few hard pieces of cosmological information have emerged from x-ray astronomy. Specifically, if there is a large amount of intergalactic gas, its temperature must be a few times $10^{8} \mathrm{~K}$. Furthermore, there is a substantial mass of hot gas associated with rich clusters of galaxies and possibly with superclusters。

Also, there are aspects of x-ray astronomy that may eventually yield cosmological information. Active galaxies (at least Seyferts) and rich clusters of galaxies are $x$-ray sources as a class and also, a large number of $\mathrm{x}$-ray sources exist with $\mathrm{Lx} / \mathrm{Lopt}>10$ and are not yet identified. Iron line emission is seen in the $x$-radiation from the cluster sources. Furthermore, the diffuse $x-r a y$ backgrounds is not yet accounted for.

The prospects for extending the present results are very good. The results so far have been obtained with very modest instruments compared to what we know is possible. HEAO-1 recently launched by NASA is the first spacecraft that carries substantially more sensitive instruments. The experiments on HEAO-1 will extend the source lists by at least a factor of 5, will extend the spectral coverage both at low and high energy range, and will allow for more precise locations and spectral data than has been possible to date. However, it is likely to be HEAO B, scheduled for launch during Fall 1978, which may provide major advances in cosmology. The HEAO-B instrument comprises a large focussing $x$-ray telescope and four focal plane instruments。 The telescope is of quality comparable to that of ordinary optical telescopes -the angular resolution over the central few arc minutes will be 2-3" and the net field of view will be about one degree. The power of this instrument comes from its great sensitivity, which derives from the simple fact that the mirror concentrates the flux incident on its 1000 $\mathrm{cm}^{2}$ aperture onto a resolution element of only $30 \mu \mathrm{m}$ diameter. The result is that background is effectively eliminated entirely and sources whose strength is only $10^{-5}$ to $10^{-6}$ photons $/ \mathrm{cm}^{-2}$ should be detectable. This is between $10^{-3}$ and $10^{-4}$ of the current faintest detectable sources. This sensitivity is so great that the question of the diffuseness of the x-ray background can be resolved unambiguously. Seyfert galaxies and clusters of galaxies can be seen to such great distances that evolutionary effects must become evident. With positional accuracy of $1 "$, optical and radio identifications can be made 
independent of assumptions regarding the nature of the object. In fact, it can be demonstrated that the ultimate limit to extracting cosmology from certain of the $\mathrm{x}$-ray data may be the inability to detect the visible emission from the objects. However, our experience in situations where the quality of observational data is vastly improved is that entirely novel results emerge. By definition, it is impossible to predict where this might occur.

I have been aided significantly in the preparation of this paper by discussions with Drs. William Forman, Riccardo Giacconi, Stephen Murray, Daniel Schwartz, and Harvey Tananbaum of the Center for Astrophysics.

Bahcall, J.N., Bahcall, N.A., Murray, S.S., Schmidt, M。: 1975, "Ap.J. (Let.t.)", 199, L9.

Bowyer, C.S., Byram, E.T., Chubb, T.A., and Friedman, H.: 1964, "Nature", $201,1307$.

Byram, E.T., Chubb, T॰A., Friedman, H.: 1966, "Science", 152:66.

Cooke, B.A., Ricketts, M.J., Maccacaro, T., Pye, J.P., Elvis, M., Watson, M.G., Griffiths, R.E., Pounds, K.A., McHardy, I., Maccagni, D。, Seward, F., Page, C.G., and Turner, M.J.L.: 1977, submitted to "Mon. Not. K. Astro. Soc.".

Cowsik, R., and Kobetich, E.J.: 1972, "Astrophys. J。", 177, 585. Culhane, L.: 1977, to be published in "Quart. Journ,, Astron. Soc." . Field, G.B., Perrenod, S.C.: 1977, "Ap.J." In Press. Forman, W., Jones, C., Cominsky, L., Julien, P., Murray, S., Peters, G。, Tananbaum, H., and Giacconi, R.: 1977, submitted to "Astrophys. J. (Supp1)".

Giacconi, R., Gursky, H., Faolini, Fo, Rossi, B.: 1962, "Phys. Rev. Lett.", 9:439.

Giacconi, R., Murray, So, Cursky, H。, Kellogg, E., Schreier, E., Matilsky, T., Koch, D., Tananbaum, ‥: 1974, "Ap.J. Suppl.", 27, No. 237,37

Gursky, H., and Schwartz, D.: 1977, "Ann. Rev. Astron。Astrophys.", 15,541 .

Gursky, $\mathrm{H}_{0}$, Solinger, A., Kellogg, E., Murray, S., Tananbaum, H., Giacconi, R., Cavaliere, A.: 1972, "Ap. J. (Lett.)", 173, L99.

Horstman, H.M., Cavallo, G。, Moretti-Horstman, E.: 1975, "Riv. Nuovo Cimento", 5:255.

Hoyle, F.: 1963, "Astrophys.J.", 137, 993。

Jones, C., and Forman, W.: 1977, sublitted to "Astrophys.J。"

Kellogg, E。, Murray, S., Giacconi, R., Tananbaum, H., Gursky, Fi॰ : 3973, "Ap.J.(Letto)", 185, L13.

Murray, S., Forman, W., Jones, C., and Giacconi, R.: 1977, subritted to "Astrop!y.J. (Lett)".

Schwartz, D.: 1974, "Int. Confo on X-rays in Space", Univ, of calgary, Calgary, Alberta.

Schwartz, D.: 1977, "Private Communication"。

Schwartz, D.A., Murray, S.S., Gursky, H。, 1976。: "Ap.J.", 204, 315。

Tananbaum, H., Peters, G., Forman, W., Giacconi, R., Jones, C., and Avni, Y.: submitted to "Astrophys。J。(Lett)"。 


\section{DISCUSSION}

Ostriker: Concerning your remark on the contribution of gas to the total mass of clusters, the gas contributes only about $10 \%$ of the total mass which is determined by dynamical arguments.

Gursky: Where is all the hidden mass?

Ostriker: We do not care. The mass is determined from dynamical arguments.

SiZk: Two comments and a question. The supercluster X-ray sources, if real and thermal, should be very extended and may therefore show up when comparing observations with different fields of view. The X-ray luminosity-velocity dispersion correlation may largely be due to the richness correlation, if velocity dispersions are even a very weak function of richness. Would you comment on the present observational status and relevance to cosmology of the hard X-ray background above $100 \mathrm{keV}$ ?

Gursky: We know very 1ittle about the sky above $100 \mathrm{keV}$. The situation is similar to what was known in the $1-10 \mathrm{keV}$ range in 1964; namely we see a few galactic sources in this energy range and see a background of radiation. In fact we have more information at 50-100 MeV than we do at around $100 \mathrm{keV}$.

Morton: I could imagine several chains and at least one large area clear of sources on the 4 th Uhuru map. Is there any reality to these features?

Gursky: The sky coverage by Uhuru was highly nonuniform because of the necessity of studying specific galactic $X$-ray sources (e.g. Hercules $\mathrm{X}-1$ ) for very long intervals.

Zasov: What part of X-ray background could originate in the vicinity of the Galaxy?

Gursky: A very small amount can be attributed to the Galaxy. Away from the Galactic centre, the contribution is only a few per cent of the diffuse background and does not extend more than a few degrees from the galactic plane.

Culhane: Regarding Dr Silk's comment on possible differences in detector fields of view as a possible explanation of the inability of the Arie1 $\mathrm{V}$ and 0SO-8 detectors to see the Uhuru superclusters, I would point out that the Ariel and OSO detectors have $3.5^{\circ}$ and $5^{\circ}$ circular fields of view, which are very comparable to the supercluster sizes and it is therefore most unlikely that these instruments would have failed to detect these sources at the Uhuru level. I would also comment on Dr Gursky's suggestion on possible systematic differences between data from different experiments; I know of no case in $\mathrm{X}-\mathrm{ray}$ astronomy where 
differences of between 3 and 6 times have later been proved due to systematic error.

Gursky: I understand Dr Culhane's point regarding the failure of Ariel 5 to confirm the superclusters. However, it is very unlikely that six Uhuru sources could be spurious. This would imply that most of the unidentified sources in the $4 \mathrm{U}$ catalogue (many of which also appear in the $2 \mathrm{~A}$ catalogue) are also spurious. Fortunately, new satellite data will resolve the question within the year.

KeZlogg: Regarding the existence of $\mathrm{X}$-ray sources in superclusters, if we accept the existence of the large scale structures reported at this conference by Einasto and Tifft as superclusters, then there are luminous $\mathrm{X}$-ray sources in these superclusters. In the Coma supercluster we see the X-ray sources associated with Abe11 1656 and Abe11 1367. In the Perseus supercluster we see Abe11 426 and Abe11 262 and perhaps others.

Gott: Regarding the effect of gas on the validity of N-body simulations, the models should give reasonable results if the total mass is distributed in a similar way to the galaxies. In Coma and other great clusters the gas and galaxies are similarly distributed. The cooling time of the gas is longer than the Hubble time, so this gas has suffered no significant dissipation relative to the galaxies. If most of the mass in the great clusters is in dark stellar halos of galaxies then the two-body relaxation effects in rich clusters will be less than in the N-body simulations.

de Vaucouleurs: Has a search been made for an enhancement of the diffuse $\mathrm{X}$-ray backgroun in the supergalactic plane?

Gursky: We attempted with the Uhuru data to construct a map in order to search for such large scale features; however, we ran into systematic differences of $\sim 5 \%$ from day to day which made it impossible to do so.

Zeldovich: What gas temperature is needed to make a diffuse intergalactic gas observable at $\mathrm{X}$-ray wavelengths?

Gursky: It is very difficult to be certain that one has observed the diffuse emission from hot intergalactic gas. One could only attribute the background to this mechanism if all other possibilities have been eliminated. It is probably best to proceed from observations of regions where the gas is known to be clumped as in clusters or superclusters.

Sunyaev: I want to mention that observations of fluctuations of the intensity of the X-ray background are approximately 4 times more informative (at the same level of $\Delta I / I$ ) than the observations in the microwave waveband. This is due to the we11-known Compton-Getting effect, and is connected with different spectral indices $J_{\nu} \propto \nu \alpha ; \alpha=2$ for the Rayleigh Jeans spectral region in the microwave waveband and $\alpha=-1$ in the $\mathrm{X}$-ray band. For example, in the case of motion of the Earth in the 
background field with velocity $\mathrm{v} \ll \mathrm{c}$

$$
\Delta I_{\nu} / I_{\nu}=(3-\alpha) \mathrm{v} / \mathrm{c} \cos \theta \text {. }
$$

It is obvious that observations in the microwave band are now more sensitive than in the X-ray band. However, it is very pleasant to know about this factor for future observations.

van der Laan: I understood you to say that between 20 to $40 \%$ of the $\mathrm{X}$-ray background in the 4-10 keV window may be contributed by the two known categories of extragalactic sources, viz. clusters and Seyfert galaxies. For the unidentified sources (probably unidentified only because of inadequate positional accuracy) we have no information at all on their X-ray luminosity function. If they have a z-dependent population evolution comparable to strong radio sources, they may contribute the remainder of the background. Do you have any reason to rule this out?

Gursky: No reason at all. In fact, Silk in 1965 developed the formulae for the contributions to the diffuse X-ray background from a population of sources which evolved as did the quasars. 